expression levels of large numbers of gene targets from microarray experiments. The inclusion of individual internal standards for large-scale experiments would be completely impractical.

In addition, we would also like to clarify that for the $\mathrm{LUX}^{\mathrm{TM}}$ primer technology from Invitrogen, it is possible to run a melting curve to examine any erroneous amplification since the fluorescence stays attached to the PCR product. This melting curve is run under the same conditions that one would use for a SYBR ${ }^{\circledR}$ Green I melting curve. Therefore, the presence of a single PCR product would likely be detected without the need to run an agarose gel, contrary to the statement in our original paper.

Address correspondence to Juan F. Medrano, Dept. of Animal Science,University of California Davis, One Shields Ave., Davis, CA 95616-8521, USA. e-mail: JFMedrano@UCDavis.edu

\title{
Correction: Facile whole-body imaging of internal fluorescent tumors in mice with an LED flashlight
}

doi 10.2144/000112055

An error appeared in Yang et al. (BioTechniques 39:170-172, August 2005; doi 10.2144/000112093) in the article entitled "Facile whole-body imaging of internal fluorescent tumors in mice with an LED flashlight." On page 170 , paragraph 4 should read as follows:

It is reported here that a blue LED flashlight (LDP LLC, Woodcliff Lake, NJ, USA; www.maxmax. com/OpticalProducts.htm) with the excitation D470/40× filter (midpoint wavelength peak of $470 \mathrm{~nm}$ ) and the OG515 Long Pass emission filter (Chroma Technology, Rockingham, VT, USA) for viewing could be used for whole-body imaging of mice with GFP and red fluorescent protein (RFP)-expressing tumors growing in or on internal organs (2).

The authors regret these oversights. The corrected PDF version of the article is available at www.BioTechniques.com. 